Commun. Korean Math. Soc. 27 (2012), No. 2, pp. 347-356

http://dx.doi.org/10.4134/CKMS.2012.27.2.347

\title{
A LOCAL FIXED POINT THEOREM ON FUZZY METRIC SPACES
}

\author{
Shaban Sedghi, Ishak Altun, and Nabi Shobe
}

\begin{abstract}
In this paper, we present a common fixed point theorem for multivalued maps on $M$-complete fuzzy metric spaces. Also, the single valued case and an illustrative example are given
\end{abstract}

\section{Introduction}

There exists considerable literature of fixed point theory dealing with results on fixed or common fixed points in fuzzy metric space (e.g. [1]-[13], [15], [18][20]). Almost all of these papers, the results have been given for single valued mappings. In the present paper, we give a local common fixed point theorem for multivalued and single valued maps on $M$-complete fuzzy metric spaces. In addition, we give an illustrative example.

For the sake of completeness, we briefly recall some notions from the theory of fuzzy metric spaces.

Definition 1 ([17]). A binary operation $*:[0,1] \times[0,1] \rightarrow[0,1]$ is called a continuous $t$-norm if $([0,1], *)$ is an abelian topological monoid with the unit 1 such that $a * b \leq c * d$ whenever $a \leq c$ and $b \leq d$ for all $a, b, c, d \in[0,1]$.

Two typical examples of continuous $t$-norms are $a * b=a b$ and $a * b=$ $\min \{a, b\}$.

Definition 2 ([6]). A fuzzy metric space (in the sense of George and Veeramani) is a triple $(X, M, *)$, where $X$ is a nonempty set, $*$ is a continuous $t$-norm and $M$ is a fuzzy set on $X^{2} \times(0, \infty)$, satisfying the following properties:

$(\mathrm{GV}-1) \quad M(x, y, t)>0, \forall t>0$,

$(\mathrm{GV}-2) M(x, x, t)=1, \forall t>0$ and if $M(x, y, t)=1$ for some $t>0$, then $x=y$,

$(\mathrm{GV}-3) M(x, y, t)=M(y, x, t), \forall x, y \in X$ and $t>0$,

$(\mathrm{GV}-4) M(x, y, \cdot):(0, \infty) \rightarrow[0,1]$ is continuous $\forall x, y \in X$,

$(\mathrm{GV}-5) M(x, z, t+s) \geq M(x, y, t) * M(y, z, s), \forall x, y, z \in X, \forall t, s>0$.

Received November 2, 2010; Revised November 19, 2010.

2010 Mathematics Subject Classification. Primary 54H25; Secondary 47H10.

Key words and phrases. fixed point, fuzzy metric space, multivalued map.

(C)2012 The Korean Mathematical Society 
Definition $3([6])$. Let $(X, M, *)$ be a fuzzy metric space. A sequence $\left\{x_{n}\right\}$ in $X$ is called a $M$-Cauchy sequence, if for each $r \in(0,1)$ and $t>0$ there exists $n_{0} \in \mathbb{N}$ such that $M\left(x_{n}, x_{m}, t\right)>1-r$ for all $m, n \geq n_{0}$. A sequence $\left\{x_{n}\right\}$ in a fuzzy metric space $(X, M, *)$ is said to be convergent to $x \in X$ if $\lim _{n \rightarrow \infty} M\left(x_{n}, x, t\right)=1$ for all $t>0$. A fuzzy metric space $(X, M, *)$ is called $M$-complete if every $M$-Cauchy sequence is convergent.

Let $(X, M, *)$ be a fuzzy metric space. For $t>0$, the open ball $B(x, r, t)$ with center $x \in X$ and radius $r(0<r<1)$ is defined by

$$
B(x, r, t)=\{y \in X: M(x, y, t)>1-r\}
$$

and the closed ball $B[x, r, t]$ is defined by

$$
B[x, r, t]=\{y \in X: M(x, y, t) \geq 1-r\} .
$$

Let $\tau$ be the set of all $A \subset X$ with $x \in A$ if and only if there exist $t>0$ and $0<r<1$ such that $B(x, r, t) \subset A$. Then $\tau$ is a topology on $X$ (induced by the fuzzy metric $M$ ). This topology is Hausdorff and first countable. A subset $A$ of $X$ is said to be $F$-bounded if there exist $t>0$ and $0<r<1$ such that $M(x, y, t)>1-r$ for all $x, y \in A$.

Example 1. Let $X=\mathbb{R}, a * b=a b$ for all $a, b \in[0,1]$ and

$$
M(x, y, t)=\frac{t}{t+|x-y|}
$$

for all $x, y \in X$ and $t \in(0, \infty)$, then $(X, M, *)$ is a fuzzy metric space.

Lemma $1([7])$. Let $(X, M, *)$ be a fuzzy metric space. Then $M(x, y, t)$ is non-decreasing with respect to $t$, for all $x, y$ in $X$.

Definition 4. Let $(X, M, *)$ be a fuzzy metric space. $M$ is said to be continuous on $X^{2} \times(0, \infty)$ if

$$
\lim _{n \rightarrow \infty} M\left(x_{n}, y_{n}, t_{n}\right)=M(x, y, t),
$$

whenever a sequence $\left\{\left(x_{n}, y_{n}, t_{n}\right)\right\}$ in $X^{2} \times(0, \infty)$ converges to a point $(x, y, t) \in$ $X^{2} \times(0, \infty)$, i.e.

$$
\lim _{n \rightarrow \infty} M\left(x_{n}, x, t\right)=\lim _{n \rightarrow \infty} M\left(y_{n}, y, t\right)=1
$$

and

$$
\lim _{n \rightarrow \infty} M\left(x, y, t_{n}\right)=M(x, y, t)
$$

Lemma 2. Let $(X, M, *)$ be a fuzzy metric space. Then $M$ is a continuous function on $X^{2} \times(0, \infty)$.

Proof. See [7] and Proposition 1 of [16].

Let $\Phi$ be the set of all strictly increasing and continuous functions $\phi$ : $(0,1] \longrightarrow(0,1]$ such that $\phi(s)>s$ for every $s \in(0,1)$. 
Example 2. Let $\phi:(0,1] \longrightarrow(0,1]$ be a function defined by $\phi(t)=\sqrt{t}$. Then it is clear that $\phi \in \Phi$.

Let $(X, M, *)$ be a fuzzy metric space and let $A, B \subseteq X$ be two nonempty sets. Define

$$
\delta_{M}(A, B, t)=\inf \{M(x, y, t): x \in A, y \in B\}
$$

and

$$
\mathcal{M}(A, B, t)=\sup \{M(x, y, t): x \in A, y \in B\}
$$

for all $t>0$. In particular, if $B=\{b\}$, then

$$
\mathcal{M}(A, B, t)=\sup \{M(x, b, t): x \in A\} .
$$

\section{Main result}

Theorem 3. Let $(X, M, *)$ be a $M$-complete fuzzy metric space such that $a * b \geq$ $a b$ for every $a, b \in[0,1]$. Let $x_{0} \in X, 0<r<1$ with $F, G: B\left[x_{0}, 1-r, t\right] \rightarrow$ $C(X)$ (the family of all nonempty closed subsets of $X$ ). Suppose, for all $x, y \in$ $B\left[x_{0}, 1-r, t\right], F x, G y$ are $F$-bounded and

$$
\delta_{M}(F x, G y, t) \geq \phi(\min \{M(x, y, t), \mathcal{M}(x, F x, t), \mathcal{M}(y, G y, t)\}) .
$$

Also we assume that the following conditions are satisfied: for all $t>0$

$$
\mathcal{M}\left(x_{0}, F x_{0}, t\right)>\frac{r}{\phi(r)}
$$

and

$$
\prod_{i=n}^{\infty} \phi^{i}\left(\frac{r}{\phi(r)}\right) \geq \phi^{n}(r)
$$

for $n \in \mathbb{N}$, where $\phi \in \Phi$.

Then there exists $x \in B\left[x_{0}, 1-r, t\right]$ with $x \in F x$ and $x \in G x$.

Proof. From (2.2) we can choose, for all $t>0, x_{1} \in F x_{0}$ with

$$
M\left(x_{0}, x_{1}, t\right)>\frac{r}{\phi(r)} .
$$

Thus $M\left(x_{0}, x_{1}, t\right)>\frac{r}{\phi(r)}>r=1-(1-r)$, hence $x_{1} \in B\left[x_{0}, 1-r, t\right]$. Now we have

$$
\phi\left(M\left(x_{0}, x_{1}, t\right)\right)>\phi\left(\frac{r}{\phi(r)}\right)
$$

(this is possible from (2.4) and the fact that $\phi$ is strictly increasing). Since $x_{0}, x_{1} \in B\left[x_{0}, 1-r, t\right]$ by $(2.1)$ we have

$$
\delta_{M}\left(F x_{0}, G x_{1}, t\right) \geq \phi\left(\min \left\{M\left(x_{0}, x_{1}, t\right), \mathcal{M}\left(x_{0}, F x_{0}, t\right), \mathcal{M}\left(x_{1}, G x_{1}, t\right)\right\}\right) .
$$

Now, choose $x_{2} \in G x_{1}$ so that

$$
\begin{aligned}
M\left(x_{1}, x_{2}, t\right) & \geq \delta_{M}\left(F x_{0}, G x_{1}, t\right) \\
& \geq \phi\left(\min \left\{M\left(x_{0}, x_{1}, t\right), \mathcal{M}\left(x_{0}, F x_{0}, t\right), \mathcal{M}\left(x_{1}, G x_{1}, t\right)\right\}\right) \\
& \geq \phi\left(\min \left\{M\left(x_{0}, x_{1}, t\right), M\left(x_{1}, x_{2}, t\right)\right\}\right) .
\end{aligned}
$$


Hence for all $t>0$, we have

$$
\begin{aligned}
M\left(x_{1}, x_{2}, t\right) & \geq \phi\left(M\left(x_{0}, x_{1}, t\right)\right) \\
& \geq \phi\left(\frac{r}{\phi(r)}\right) .
\end{aligned}
$$

Therefore we have

$$
\phi\left(M\left(x_{1}, x_{2}, t\right)\right)>\phi^{2}\left(\frac{r}{\phi(r)}\right) .
$$

Since

$$
\begin{aligned}
M\left(x_{0}, x_{2}, t\right) & \geq M\left(x_{0}, x_{1}, \frac{t}{2}\right) * M\left(x_{1}, x_{2}, \frac{t}{2}\right) \\
& \geq \frac{r}{\phi(r)} * \phi\left(\frac{r}{\phi(r)}\right) \\
& \geq \frac{r}{\phi(r)} \phi(r) \\
& =r
\end{aligned}
$$

then we have $x_{2} \in B\left[x_{0}, 1-r, t\right]$. Again, choose $x_{3} \in F x_{2}$ so that (since $x_{1}, x_{2} \in B\left[x_{0}, 1-r, t\right]$ we can use the inequality $(2.1)$ )

$$
\begin{aligned}
M\left(x_{3}, x_{2}, t\right) & \geq \delta_{M}\left(F x_{2}, G x_{1}, t\right) \\
& \geq \phi\left(\min \left\{M\left(x_{2}, x_{1}, t\right), \mathcal{M}\left(x_{2}, F x_{2}, t\right), \mathcal{M}\left(x_{1}, G x_{1}, t\right)\right\}\right) \\
& \geq \phi\left(\min \left\{M\left(x_{2}, x_{1}, t\right), M\left(x_{2}, x_{3}, t\right)\right\}\right) .
\end{aligned}
$$

Now, from (2.6), we have

$$
M\left(x_{3}, x_{2}, t\right) \geq \phi\left(M\left(x_{1}, x_{2}, t\right)\right) \geq \phi^{2}\left(\frac{r}{\phi(r)}\right)
$$

and so we have

$$
\phi\left(M\left(x_{3}, x_{2}, t\right)\right) \geq \phi^{3}\left(\frac{r}{\phi(r)}\right) .
$$

Also, for all $t>0$, since

$$
\begin{aligned}
M\left(x_{0}, x_{3}, t\right) & \geq M\left(x_{0}, x_{1}, \frac{t}{3}\right) * M\left(x_{1}, x_{2}, \frac{t}{3}\right) * M\left(x_{2}, x_{3}, \frac{t}{3}\right) \\
& \geq \frac{r}{\phi(r)} \phi\left(\frac{r}{\phi(r)}\right) \phi^{2}\left(\frac{r}{\phi(r)}\right) \\
& \geq \frac{r}{\phi(r)} \prod_{i=1}^{\infty} \phi^{i}\left(\frac{r}{\phi(r)}\right) \\
& \geq \frac{r}{\phi(r)} \phi(r)=r
\end{aligned}
$$

we have $x_{3} \in B\left[x_{0}, 1-r, t\right]$. 
Continuing this way we can obtain a sequence $\left\{x_{n}\right\} \subseteq B\left[x_{0}, 1-r, t\right]$ such that $x_{2 n+2} \in G x_{2 n+1}$ and $x_{2 n+1} \in F x_{2 n}$ for $n \in\{0,1, \ldots\}$ and

$$
M\left(x_{n}, x_{n+1}, t\right)>\phi^{n}\left(\frac{r}{\phi(r)}\right) \text {. }
$$

Next we show that $\left\{x_{n}\right\}$ is a $M$-Cauchy sequence. Notice for all $t>0$ and $0<k<1$ we can choose $t_{0}>0$ such that $t=\sum_{i=0}^{\infty} k^{i} t_{0}$. Hence using (2.3) and (2.7) we have

$$
\begin{aligned}
& M\left(x_{n}, x_{m}, t\right) \\
= & M\left(x_{n}, x_{m}, \sum_{i=0}^{\infty} k^{i} t_{0}\right) \\
\geq & M\left(x_{n}, x_{m}, \sum_{i=n}^{m-1} k^{i} t_{0}\right) \\
\geq & M\left(x_{n}, x_{n+1}, k^{n} t_{0}\right) * M\left(x_{n}, x_{n+2}, k^{n+1} t_{0}\right) * \cdots * M\left(x_{n}, x_{m}, k^{m-1} t_{0}\right) \\
\geq & \phi^{n}\left(\frac{r}{\phi(r)}\right) \phi^{n+1}\left(\frac{r}{\phi(r)}\right) \cdots \phi^{m-1}\left(\frac{r}{\phi(r)}\right) \\
\geq & \prod_{i=n}^{\infty} \phi^{i}\left(\frac{r}{\phi(r)}\right) \geq \phi^{n}(r) \longrightarrow 1
\end{aligned}
$$

and so $\left\{x_{n}\right\}$ is a $M$-Cauchy sequence. Since $X$ is $M$-complete, then there exists $x \in B\left[x_{0}, 1-r, t\right]$ with $x_{n} \rightarrow x$. It remains to show $x \in F x$ and $x \in G x$.

Let $\mathcal{M}(x, F x, t)<1$ for all $t>0$. Since $x_{2 n-1}, x \in B\left[x_{0}, 1-r, t\right]$ we can use the inequality (2.1), then we have

$$
\begin{aligned}
& \delta_{M}\left(F x, G x_{2 n-1}, t\right) \\
\geq & \phi\left(\min \left\{M\left(x, x_{2 n-1}, t\right), \mathcal{M}(x, F x, t), \mathcal{M}\left(x_{2 n-1}, G x_{2 n-1}, t\right)\right)\right. \\
\geq & \phi\left(\min \left\{M\left(x, x_{2 n-1}, t\right), \mathcal{M}(x, F x, t), t\right), M\left(x_{2 n-1}, x_{2 n}, t\right)\right) .
\end{aligned}
$$

Now taking limit inferior to $n \rightarrow \infty$ we have, for all $t>0$,

$$
\liminf _{n \rightarrow \infty} \delta_{M}\left(F x, G x_{2 n-1}, t\right) \geq \phi(\mathcal{M}(x, F x, t)) .
$$

On the other hand, for $0<\varepsilon<t$, we have

$$
\begin{aligned}
\mathcal{M}(x, F x, t) & \geq M\left(x, x_{2 n}, \varepsilon\right) * \mathcal{M}\left(x_{2 n}, F x, t-\varepsilon\right) \\
& \geq M\left(x, x_{n}, \varepsilon\right) * \delta_{M}\left(G x_{2 n-1}, F x, t-\varepsilon\right)
\end{aligned}
$$

and so, taking limit inferior to $n \rightarrow \infty$ and using (2.8), we have

$$
\begin{aligned}
\mathcal{M}(x, F x, t) & \geq \liminf _{n \rightarrow \infty} \delta_{M}\left(F x, G x_{2 n-1}, t-\varepsilon\right) \\
& \geq \phi(\mathcal{M}(x, F x, t-\varepsilon)) .
\end{aligned}
$$

Taking $\varepsilon \rightarrow 0$ we have

$$
\mathcal{M}(x, F x, t) \geq \phi(\mathcal{M}(x, F x, t)),
$$


which is a contradiction ( since $\phi(s)>s$ for $0<s<1$ and $\mathcal{M}(x, F x, t)>0$ ). Thus $\mathcal{M}(x, F x, t)=1$ for some $t>0$. Then $x \in \overline{F x}=F x$.

Similarly, we obtain $\mathcal{M}(x, G x, t)=1$ for some $t>0$, so $x \in G x$.

Now we give an illustrative example.

Example 4. Let $X=(0, \infty), a * b=a b$ and

$$
M(x, y, t)=\frac{\min \{x, y\}}{\max \{x, y\}}, \forall t>0 .
$$

Then $(X, M, *)$ is a $M$-complete fuzzy metric space (see [14]). Let $x_{0}=\frac{1}{2}$ and $r=\frac{1}{3}$, then

$$
\begin{aligned}
B\left[x_{0}, 1-r, t\right] & =B\left[\frac{1}{2}, \frac{2}{3}, t\right] \\
& =\left\{y \in X: M\left(\frac{1}{2}, y, t\right) \geq \frac{1}{3}\right\} \\
& =\left[\frac{1}{6}, \frac{3}{2}\right] .
\end{aligned}
$$

Now let $F, G: B\left[x_{0}, 1-r, t\right] \rightarrow C(X)$,

$$
F x=G x=\left\{\begin{array}{cc}
{[1, \sqrt{x}] \quad, \quad x \geq 1} \\
\sqrt{x} \quad, \quad x<1
\end{array}\right.
$$

and let $\phi(t)=\sqrt{t}$, then it is clear that $\phi \in \Phi$. Also $F x$ and $G y$ are $F$-bounded,

$$
\begin{aligned}
\mathcal{M}\left(x_{0}, F x_{0}, t\right) & =\mathcal{M}\left(\frac{1}{2}, \frac{1}{\sqrt{2}}, t\right) \\
& =M\left(\frac{1}{2}, \frac{1}{\sqrt{2}}, t\right)=\frac{\sqrt{2}}{2}>\frac{\sqrt{3}}{3}=\frac{\frac{1}{3}}{\phi\left(\frac{1}{3}\right)}=\frac{r}{\phi(r)}
\end{aligned}
$$

and

$$
\prod_{i=n}^{\infty} \phi^{i}\left(\frac{r}{\phi(r)}\right)=\prod_{i=n}^{\infty} r^{\frac{1}{2^{i+1}}}=r^{\sum_{i=n}^{\infty} \frac{1}{2^{i+1}}}=r^{\frac{1}{2^{n}}}=\phi^{n}(r) .
$$

Finally, we show that $(2.1)$ is satisfied for all $x, y \in B\left[x_{0}, 1-r, t\right]=\left[\frac{1}{6}, \frac{3}{2}\right]$. For this we consider the following cases: 
Case 1. if $x, y \in\left[1, \frac{3}{2}\right]$ and $x>y$, then

$$
\begin{aligned}
\delta_{M}(F x, G y, t) & =\inf \{M(a, b, t): a \in F x=[1, \sqrt{x}], b \in G y=[1, \sqrt{y}]\} \\
& =\inf \left\{\frac{\min \{a, b\}}{\max \{a, b\}}: a \in F x=[1, \sqrt{x}], b \in G y=[1, \sqrt{y}]\right\} \\
& =\frac{1}{\sqrt{x}} \\
& =\phi\left(\inf \left\{\frac{\min \{x, c\}}{\max \{x, c\}}: c \in F x=[1, \sqrt{x}]\right\}\right) \\
& =\phi(\inf \{M(x, c, t): c \in F x=[1, \sqrt{x}]\}) \\
& =\phi(\mathcal{M}(x, F x, t)) \\
& \geq \phi(\min \{M(x, y, t), \mathcal{M}(x, F x, t), \mathcal{M}(y, G y, t)\}) .
\end{aligned}
$$

Case 2. if $x \in\left[1, \frac{3}{2}\right]$ and $y \in\left[\frac{1}{2}, 1\right)$, then

$$
\begin{aligned}
\delta_{M}(F x, G y, t) & =\inf \{M(a, b, t): a \in F x=[1, \sqrt{x}], b \in G y=\sqrt{y}\} \\
& =\inf \left\{\frac{\min \{a, b\}}{\max \{a, b\}}: a \in F x=[1, \sqrt{x}], b \in G y=\sqrt{y}\right\} \\
& =\frac{\sqrt{y}}{\sqrt{x}} \\
& =\sqrt{\frac{\min \{x, y\}}{\max \{x, y\}}} \\
& =\phi(M(x, y, t)) \\
& \geq \phi(\min \{M(x, y, t), \mathcal{M}(x, F x, t), \mathcal{M}(y, G y, t)\}) .
\end{aligned}
$$

Case 3. if $x, y \in\left[\frac{1}{2}, 1\right)$ and $x>y$, then

$$
\begin{aligned}
\delta_{M}(F x, G y, t) & =\inf \{M(a, b, t): a \in F x=\sqrt{x}, b \in G y=\sqrt{y}\} \\
& =\frac{\sqrt{y}}{\sqrt{x}} \\
& =\sqrt{\frac{\min \{x, y\}}{\max \{x, y\}}} \\
& =\phi(M(x, y, t)) \\
& \geq \phi(\min \{M(x, y, t), \mathcal{M}(x, F x, t), \mathcal{M}(y, G y, t)\})
\end{aligned}
$$

Therefore conditions of Theorem 3 are satisfied, thus $F$ and $G$ have a common fixed point in $B\left[x_{0}, 1-r, t\right]=\left[\frac{1}{6}, \frac{3}{2}\right]$.

We can have the following corollaries.

Corollary 1. Let $(X, M, *)$ be a $M$-complete fuzzy metric space such that $a * b \geq a b$ for every $a, b \in[0,1]$. Let $x_{0} \in X, 0<r<1$ with $F, G: B\left[x_{0}, 1-\right.$ 
$r, t] \rightarrow X$. Suppose, for all $x, y \in B\left[x_{0}, 1-r, t\right]$

$$
M(F x, G y, t) \geq \phi(\min \{M(x, y, t), M(x, F x, t), M(y, G y, t)\}) .
$$

Also we assume that the following conditions are satisfied: for all $t>0$

$$
M\left(x_{0}, F x_{0}, t\right)>\frac{r}{\phi(r)}
$$

and

$$
\prod_{i=n}^{\infty} \phi^{i}\left(\frac{r}{\phi(r)}\right) \geq \phi^{n}(r)
$$

for $n \in \mathbb{N}$, where $\phi \in \Phi$.

Then there exists a unique $x \in B\left[x_{0}, 1-r, t\right]$ with $x=F x=G x$.

Proof. By Theorem 3, it is enough prove that $x$ is unique.

Let $y$ be another common fixed point of $F$ and $G$, that is $y=F y=G y$, then we have

$$
\begin{aligned}
M(F x, G y, t) & \geq \phi(\min \{M(x, y, t), M(x, F x, t), M(y, G y, t)\}) \\
& =\phi(M(x, y, t))>M(x, y, t),
\end{aligned}
$$

which is a contradiction. Therefore $F$ and $G$ have a unique common fixed point in $B\left[x_{0}, 1-r, t\right]$.

Corollary 2. Let $(X, M, *)$ be a $M$-complete fuzzy metric space such that $a * b \geq a b$ for every $a, b \in[0,1]$. Let $x_{0} \in X, 0<r<1$ with $F: B\left[x_{0}, 1-r, t\right] \rightarrow$ $X$. Suppose, for all $x, y \in B\left[x_{0}, 1-r, t\right]$

$$
M(F x, F y, t) \geq \phi(\min \{M(x, y, t), M(x, F x, t), M(y, F y, t)\}) .
$$

Also we assume that the following conditions are satisfied: for all $t>0$

$$
M\left(x_{0}, F x_{0}, t\right)>\frac{r}{\phi(r)}
$$

and

$$
\prod_{i=n}^{\infty} \phi^{i}\left(\frac{r}{\phi(r)}\right) \geq \phi^{n}(r)
$$

for $n \in \mathbb{N}$, where $\phi \in \Phi$.

Then there exists a unique $x \in B\left[x_{0}, 1-r, t\right]$ with $x=F x$.

Example 5. Let $X, *, M, x_{0}, r$ and $\phi$ be as in Example 4. Let $F: B\left[x_{0}, 1-\right.$ $r, t] \rightarrow X, F x=\sqrt{x}$, then it is clear that

$$
M\left(x_{0}, F x_{0}, t\right)=M\left(\frac{1}{2}, \frac{1}{\sqrt{2}}, t\right)=\frac{\sqrt{2}}{2}>\frac{\sqrt{3}}{3}=\frac{\frac{1}{3}}{\phi\left(\frac{1}{3}\right)}=\frac{r}{\phi(r)}
$$

and

$$
\prod_{i=n}^{\infty} \phi^{i}\left(\frac{r}{\phi(r)}\right)=\prod_{i=n}^{\infty} r^{\frac{1}{2^{i+1}}}=r^{\sum_{i=n}^{\infty} \frac{1}{2^{i+1}}}=r^{\frac{1}{2^{n}}}=\phi^{n}(r) .
$$


Also, for all $x, y \in B\left[x_{0}, 1-r, t\right]$, we have

$$
\begin{aligned}
M(F x, F y, t) & =\frac{\min \{\sqrt{x}, \sqrt{y}\}}{\max \{\sqrt{x}, \sqrt{y}\}} \\
& =\sqrt{\frac{\min \{x, y\}}{\max \{x, y\}}} \\
& =\phi(M(x, y, t)) \\
& \geq \phi(\min \{M(x, y, t), M(x, F x, t), M(y, F y, t)\}) .
\end{aligned}
$$

Therefore conditions of Corollary 2 are satisfied, thus $F$ has a unique fixed point in $B\left[x_{0}, 1-r, t\right]=\left[\frac{1}{6}, \frac{3}{2}\right]$.

Using recent ideas in the literature [2], [3], it is possible to extend our theorem to a fuzzy metric space endowed with a partial order induced by an appropriate function.

\section{References}

[1] A. Aliouche, F. Merghadi, and A. Djoudi, A related fixed point theorem in two fuzzy metric spaces, J. Nonlinear Sci. Appl. 2 (2009), no. 1, 19-24.

[2] I. Altun, Some fixed point theorems for single and multi valued mappings on ordered non-Archimedean fuzzy metric spaces, Iran. J. Fuzzy Syst. 7 (2010), no. 1, 91-96.

[3] I. Altun and D. Mihet, Ordered non-Archimedean fuzzy metric spaces and some fixed point results, Fixed Point Theory Appl. 2010 (2010), 11pages.

[4] Y. J. Cho, Fixed points in fuzzy metric spaces, J. Fuzzy Math. 5 (1997), no. 4, 949-962.

[5] J. X. Fang, On fixed point theorems in fuzzy metric spaces, Fuzzy Sets and Systems 46 (1992), no. 1, 107-113.

[6] A. George and P. Veeramani, On some result in fuzzy metric space, Fuzzy Sets and Systems 64 (1994), no. 3, 395-399.

[7] M. Grabiec, Fixed points in fuzzy metric spaces, Fuzzy Sets and Systems 27 (1988), no. $3,385-389$.

[8] V. Gregori and A. Sapena, On fixed-point theorem in fuzzy metric spaces, Fuzzy Sets and Systems 125 (2002), no. 2, 245-252.

[9] O. Hadžić and E. Pap, Fixed Point Theory in Probabilistic Metric Spaces, Kluwer Academic Publishers, Dordrecht, 2001.

[10] S. Jain, S. Jain, and L. Bahadur, Compatibility of type $(P)$ in modified intuitionisticfuzzymetric space, J. Nanlinear Sci. Appl. 3 (2010), no. 2, 96-109.

[11] D. Mihet,, On fuzzy contractive mappings in fuzzy metric spaces, Fuzzy Sets and Systems 158 (2007), no. 8, 915-921.

[12] _ Fuzzy $\psi$-contractive mappings in non-Archimedean fuzzy metric spaces, Fuzzy Sets and Systems 159 (2008), no. 6, 739-744.

[13] S. N. Mishra, S. N. Sharma, and S. L. Singh, Common fixed points of maps on fuzzy metric spaces, Internat. J. Math. Math. Sci. 17 (1994), no. 2, 253-258.

[14] V. Radu, Some remarks on the probabilistic contractions on fuzzy Menger spaces, Automat. Comput. Appl. Math. 11 (2002), no. 1, 125-131.

[15] K. P. R. Rao, A. Aliouche, and G. R. Babu, Related fixed point theorems in fuzzy metric spaces, J. Nonlinear Sci. Appl. 1 (2008), no. 3, 194-202.

[16] J. L. Rodríguez and S. Ramaguera, The Hausdorff fuzzy metric on compact sets, Fuzzy Sets and Systems 147 (2004), no. 2, 273-283.

[17] B. Schweizer and A. Sklar, Statistical metric spaces, Pacific J. Math. 10 (1960), 313-334. 
[18] S. Sedghi, N. Shobe, and I. Altun, A fixed fuzzy point for fuzzy mappings in complete metric spaces, Math. Commun. 13 (2008), no. 2, 289-294.

[19] S. Sedghi, I. Altun, and N. Shobe, Coupled fixed point theorems for contractions in fuzzy metric spaces, Nonlinear Anal. 72 (2010), no. 3-4, 1298-1304.

[20] T. Žikić-Došenović, A common fixed point theorem for compatible mappings in fuzzy metric spaces using implicit relation, Acta Math. Hungar 125 (2009), no. 4, 357-368.

ShABAN SEDGHI

Department of Mathematics

QAEMSHAHR BRANCH

ISLAMic AZAD UNIVERSiTy

Ghaemshahr P.O.Box 163, Iran

E-mail address: sedghi_gh@yahoo.com

ISHAK Altun

Department of Mathematics

Faculty of Science and ARts

KIRIKKALE UNIVERSITY

71450 Yahsihan, KiRIKKale, TuRKeY

E-mail address: ialtun@kku.edu.tr; ishakaltun@yahoo.com

Nabi Shobe

Department of Mathematics

Islamic Azad University-Babol Branch

IRAN

E-mail address: nabi_shobe@yahoo.com 